\title{
A NEW FUNCTIONAL EQUATION OF PEXIDER TYPE RELATED TO THE COMPLEX EXPONENTIAL FUNCTION
}

\author{
HIROSHI HARUKI AND THEMISTOCLES M. RASSIAS \\ Dedicated to the memory of Professor A. M. Ostrowski
}

\begin{abstract}
The purpose of this paper is to solve a new functional equation, characteristic for the complex exponential function, which contains four unknown entire functions and to solve, as an application, three further functional equations.
\end{abstract}

\section{INTRODUCTION AND STATEMENT OF THE RESULTS}

Ostrowski (see [18]) investigated functional equations related to the real exponential function in the real domain. In the present paper we shall study functional equations related to the complex exponential function in the complex domain. We first mention the log-quadratic functional equation (cf. [10], the same was suggested in [4])

$$
f(x+y) f(x-y)=f(x)^{2} f(y)^{2},
$$

where $f$ is an unknown entire function and $x, y$ are complex variables. The following theorem holds.

Theorem A. The only entire solutions of (1) are given by $f(z) \equiv 0, f(z)=$ $\exp \left(a z^{2}\right)$, and $f(z)=-\exp \left(a z^{2}\right)$, where $a$ is an arbitrary complex constant.

Next we consider the following two Cauchy equations (cf. [1, pp. 31-42] and [2, pp. 11-24]):

$$
f(x+y)=f(x)+f(y)
$$

and

$$
f(x+y)=f(x) f(y),
$$

where $f, x, y$ are as above. If we replace in (2) and (3) $x$ and $y$ by $s$ and $i t$, respectively, where $s, t$ are real variables and we take the absolute values of the resulting equalities, then we obtain the following two functional equations:

$$
|f(s+i t)|=|f(s)+f(i t)|
$$

Received by the editors March 7, 1994; originally communicated to the Proceedings of the AMS by Palle E. T. Jorgensen.

1991 Mathematics Subject Classification. Primary 39B40; Secondary 30D05.

Key words and phrases. Complex exponential function, functional equation of Pexider type, Hille's functional equation, Robinson's functional equation. 
(Robinson's functional equation; cf. [2, p. 69], [3], [9], [19]) and

$$
|f(s+i t)|=|f(s) f(i t)|
$$

(Hille's functional equation; cf. [1, p. 140], [3], [13], [14], [20]), where $f$ is an unknown entire function and $s, t$ are real variables.

In a similar way, replacing $x$ and $y$ by $s$ and it in (1) where $s, t$ are real variables and taking the absolute values of both sides of the resulting equality yield the functional equation

$$
|f(s+i t) f(s-i t)|=\left|f(s)^{2} f(i t)^{2}\right|
$$

for all real $s$ and $t$.

Now we give a generalization of this functional equation (4). We consider the following functional equation of Pexider type (cf. [1, p. 10], [2, p. 42], [5], [11]):

$$
|f(s+i t) g(s-i t)|=|h(s) k(i t)|,
$$

where $f, g, h, k$ are unknown entire functions and $s, t$ are real variables.

The purpose of this paper is to solve (5) and to give an application for the solution of three further functional equations. We first give the definition of a class of entire functions.

Definition. We denote by $\Omega$ the set of all entire functions which have power series expansions $\sum_{n=0}^{+\infty} A_{n} z^{n}$, where each $A_{n} \quad(n=0,1,2, \ldots)$ is a real constant.

For the class $\Omega$ the following proposition can be proved (we omit the easy proof).

Proposition A. For an entire function $p(z)$ to belong to the class $\Omega$ it is necessary and sufficient that $p$ satisfies $\overline{p(z)}=p(\bar{z})$ for all complex $z$.

Cautionary remark. If $p$ is a real-valued function on a subset $S$ of the $z$-plane, then

$$
|\exp (i p(z))|=1 \quad \text { for all } z \in S .
$$

This is clear from the formula $|\exp \gamma|=\exp (\operatorname{Re}(\gamma))$ for all complex $\gamma$, but we use this throughout the present paper.

We may now state our result.

Theorem 1. All systems of entire solutions of (5) are given by the following.

(i) At least one of $f, g$ is identically zero and at least one of $h, k$ is identically zero. The remaining unknown functions are arbitrary entire functions;

$$
\left\{\begin{array}{l}
f(z)=\exp (\varphi(z)) \\
g(z)=\exp \left(a z^{2}+\bar{b} z+\bar{c}-\overline{\varphi(\bar{z})}\right), \\
h(z)=l \exp \left(a z^{2}+b z+c+i p(z)\right), \\
k(z)=m \exp \left(a z^{2}+b z+c+q(z)+i r(z)\right),
\end{array}\right.
$$

where $\varphi$ is an arbitrary entire function not necessarily belonging to $\Omega, a$ is an arbitrary real constant, $b$ is an arbitrary complex constant, $c, l, m$ are arbitrary complex constants satisfying $|l m \exp c|=1$, where $p, q, r$ are arbitrary 
entire functions belonging to the class $\Omega$, and $q, r$ are odd or even functions, respectively.

\section{LEMMAS}

To prove the theorem in $\S 1$ we need the following two lemmas.

Lemma 1. All entire functions $H$ which satisfy

$$
|H(s)|=1 \text { for all real } s
$$

are given by

$$
H(z)=\exp (i p(z)), \quad \text { where } p \in \Omega .
$$

Proof. By (7) we have $H(s) \overline{H(s)}=1$, and so, by observing that $s=\bar{s}$ for all real $s$, it follows that

$$
H(s) \overline{H(\bar{s})}=1
$$

for all real numbers $s$. Since, by hypothesis, $H$ is an entire function of $z$, $\overline{H(\bar{z})}$ is also an entire function (see [7, p. 28]) and so is the product $H(z) \overline{H(\bar{z})}$.

Hence, by (8) and by the Identity Theorem,

$$
H(z) \overline{H(\bar{z})}=1
$$

holds for all complex $z$. By (9) we have

$$
H(z) \neq 0
$$

for all complex numbers $z$. By (10) and by a standard theorem (see [7, p. 192], $[12$, p. 127], and [15]) there exists an entire function $\varphi$ such that

$$
H(z)=\exp (\varphi(z))
$$

holds for all complex $z$. By (9) and (11) we obtain

$$
\exp (\varphi(z)+\overline{\varphi(\bar{z})}) \equiv 1
$$

Differentiating both sides of the above equality yields

$$
\exp (\varphi(z)+\overline{\varphi(\bar{z})})(\varphi(z)+\overline{\varphi(\bar{z})})^{\prime}=0
$$

and so

$$
\varphi(z)+\overline{\varphi(\bar{z})}=C,
$$

where $C$ is a complex constant. Let the power series of the entire function $\varphi$ be

$$
\varphi(z)=\sum_{n=0}^{+\infty} c_{n} z^{n},
$$

where the $c_{n}(n=0,1,2, \ldots)$ are complex constants. Substituting the above equality into (12) and equating the coefficients of $z^{n}(n=1,2,3, \ldots)$ in the resulting equality yield $c_{n}+\bar{c}_{n}=0 \quad(n=1,2,3, \ldots)$.

By the above equality, the $c_{n}(n=1,2,3, \ldots)$ are purely imaginary constants. Furthermore, by (11) we have

$$
H(z)=\exp \left(\sum_{n=0}^{+\infty} c_{n} z^{n}\right) .
$$


Setting here $z=0$ in (13) yields $H(0)=\exp \left(c_{0}\right)$. Setting $s=0$ in (7) and using the above equality, we get $\left|\exp \left(c_{0}\right)\right|=1$, and so we find that $c_{0}$ is also purely imaginary. Summarizing, the $c_{n}(n=0,1,2, \ldots)$ are purely imaginary. Thus, we can write $c_{n}=i a_{n}(n=0,1,2, \ldots)$, where the $a_{n}$ $(n=0,1,2, \ldots)$ are real. Hence, by $(13)$ we obtain

$$
H(z)=\exp \left(i \sum_{n=0}^{+\infty} a_{n} z^{n}\right)
$$

for all complex numbers $z$, where the $a_{n}(n=0,1,2, \ldots)$ are real numbers. Therefore, the function $p$ defined by

$$
p(z)=\sum_{n=0}^{+\infty} a_{n} z^{n}
$$

for all complex $z$ is in $\Omega$. So we have

$$
H(z)=\exp (i p(z))
$$

for all complex $z$, where $p \in \Omega$. The converse, i.e., the fact that $H(z)$ satisfies (7), is clear from (6). Q.E.D.

Lemma 2. The only entire function $K$ which satisfies

$$
|K(i t)|=1
$$

for all real $t$ is given by

$$
K(z)=\exp (q(z)+\operatorname{ir}(z)),
$$

where the functions $q, r \in \Omega$ are odd or even, respectively, but otherwise arbitrary.

Proof. Apply Lemma 1 to the entire function $K \circ \phi$, where $\phi(z)=i z$, to see that $K$ must have the desired form.

For the sake of easy understanding of the proof of the main theorem, we divide it into four steps.

\section{PROOF OF THE MAIN THEOREM}

Step 1 . We may assume that

$$
f(z) \not \equiv 0 \text { and } g(z) \not \equiv 0 .
$$

Otherwise we obtain the system of solutions (i) in Theorem 1. We show in this step that

$$
f(z) \neq 0 \text { and } g(z) \neq 0
$$

for all complex $z$ by using a method described in the two books by Aczél (see $[1$, p. 38] and [2, p. 28]). To prove that $f(z) \neq 0$ for all complex $z$, we use the method by contradiction. Assume the contrary. Then there exists a complex number $s_{0}+i t_{0} \quad\left(s_{0}, t_{0} \in \mathfrak{R}\right)$ such that $f\left(s_{0}+i t_{0}\right)=0$. Hence, by (5) we obtain $h\left(s_{0}\right) k\left(i t_{0}\right)=0$. We discuss two cases.

Case 1. The case when $h\left(s_{0}\right)=0$.

Setting $s=s_{0}$ in (5) and using the Identity Theorem yield a result contrary to $(15)$. 
Case 2. The case when $k\left(i t_{0}\right)=0$.

By a similar argument to that of Case 1 we get a contradiction. Similarly, we can prove that $g(z) \neq 0$ for all complex $z$. So (16) has been proved.

Step 2. We shall determine the unknown entire functions $f, g$. If we set $z=$ $s+i t(s, t \in \mathfrak{R})$ in $(5)$, then we have

(17) $|f(s+i t) g(s-i t)|=|f(z) g(\bar{z})|=|f(z)||g(\bar{z})|=|f(z)||\overline{g(\bar{z})}|=|f(z) \overline{g(\bar{z})}|$

for all complex numbers $z$.

By (5) and (17) we have

$$
|f(z) \overline{g(\bar{z})}|=|h(s) k(i t)|
$$

If we set

$$
\lambda(z)=f(z) \overline{g(\bar{z})},
$$

then, by (18), we obtain

$$
|\lambda(z)|=|h(s) k(i t)|
$$

for all complex numbers $z$. By (16) and (19) we obtain

$$
\lambda(z) \neq 0
$$

for all complex numbers $z$. Since $(21)$ holds, taking the logarithms of both sides of (20) yields

$$
\log |\lambda(z)|=\log |h(s)|+\log |k(i t)|
$$

for all complex $z$. Taking into account the fact that $\lambda$ is entire, applying $\frac{\partial}{\partial s}$ to both sides of the above equality, and using

$$
\frac{\partial}{\partial s} \log |f(z)|=\operatorname{Re}\left(\frac{f^{\prime}(z)}{f(z)}\right)
$$

(see $[16$, p. 55]) yield

$$
\operatorname{Re}\left(\frac{\lambda^{\prime}(z)}{\lambda(z)}\right)=\frac{\partial}{\partial s} \log |h(s)|
$$

for all complex $z$.

Applying $\frac{\partial}{\partial t}$ to both sides of (22) and using differentiation for composite functions yield

$$
\operatorname{Re}\left(i \frac{d}{d z}\left(\frac{\lambda^{\prime}(z)}{\lambda(z)}\right)\right)=0
$$

for all complex $z$. Hence, by the formula $\operatorname{Re}(i \gamma)=-\operatorname{Im}(\gamma)$, we obtain

$$
\operatorname{Im}\left(\frac{d}{d z}\left(\frac{\lambda^{\prime}(z)}{\lambda(z)}\right)\right)=0
$$

for all complex numbers $z$. Since $\frac{d}{d z}\left(\frac{\lambda^{\prime}(z)}{\lambda(z)}\right)$ is an entire function of $z$, by the above equality (see $[16$, p. 43]) we have

$$
\frac{d}{d z}\left(\frac{\lambda^{\prime}(z)}{\lambda(z)}\right)=A
$$


where $A$ is a real constant. Hence we obtain

$$
\frac{\lambda^{\prime}(z)}{\lambda(z)}=A z+b
$$

and so

$$
\lambda^{\prime}(z)=(A z+b) \lambda(z),
$$

where $b$ is a complex constant. Solving the above linear differential equation of the first order yields

$$
\lambda(z)=\exp \left(a z^{2}+b z+c\right)
$$

for all complex numbers $z$, where $a=\frac{1}{2} A(\in \mathfrak{R}$ by the fact that $A \in \mathfrak{R})$ and $b, c$ are complex constants. By (19) and (23) we obtain

$$
f(z) \overline{g(\bar{z})}=\exp \left(a z^{2}+b z+c\right)
$$

for all complex numbers $z$. Since, by (16), $f$ is an entire function which is nowhere zero, by a standard theorem there exists an entire function $\varphi$ of $z$ such that

$$
f(z)=\exp (\varphi(z))
$$

holds for all complex $z$. By (24) and (25) we have

$$
\overline{g(\bar{z})}=\exp \left(a z^{2}+b z+c-\varphi(z)\right),
$$

and so, by taking into account the fact that $a \in \mathfrak{R}(\bar{a}=a)$,

$$
g(z)=\exp \left(a z^{2}+\bar{b} z+\bar{c}-\overline{\varphi(\bar{z})}\right),
$$

where $a$ is a real constant and $b, c$ are complex constants. Thus, $f$ and $g$ are given by (25) and (26).

Step 3. We shall determine unknown entire functions $h, k$. By (20) and (23) we have

$$
|h(s) k(i t)|=\left|\exp \left(a z^{2}+b z+c\right)\right|
$$

for all complex numbers $z=s+i t \quad(s, t \in \mathfrak{R})$, where $a$ is a real constant and $b, c$ are complex constants. If we set $t=0$ in (27), then $z=s+i t$ becomes $s$. Therefore, we obtain

$$
|h(s) k(0)|=\left|\exp \left(a s^{2}+b s+c\right)\right|
$$

for all real numbers $s$. If we set

$$
H(z)=\frac{h(z) k(0)}{\exp \left(a z^{2}+b z+c\right)}
$$

for all complex numbers $z$, then, by (28), we obtain

$$
|H(s)|=1
$$

for all real $s$. Since, by (29), $H$ is an entire function of $z$, by (30) and by Lemma $1, H$ can be written as

$$
H(z)=\exp (i p(z)),
$$

where $p \in \Omega$. Hence, by (29) we have

$$
h(z) k(0)=\exp \left(a z^{2}+b z+c+i p(z)\right),
$$


where $p \in \Omega$. By $(31)$ we have $k(0) \neq 0$. So, setting $l=\frac{1}{k(0)}$ yields

$$
h(z)=l \exp \left(a z^{2}+b z+c+i p(z)\right)
$$

for all complex $z$. If we set $s=0$ in (27), then we obtain

$$
|h(0) k(i t)|=\left|\exp \left(a(i t)^{2}+b(i t)+c\right)\right|
$$

for all real $t$. If we set

$$
K(z)=\frac{h(0) k(z)}{\exp \left(a z^{2}+b z+c\right)}
$$

for all complex $z$, then, by (33), we obtain

$$
|K(i t)|=1
$$

for all real numbers $t$. Since, by (34), $K$ is an entire function of $z$, by (35) and by Lemma $2, K$ can be written as

$$
K(z)=\exp (q(z)+\operatorname{ir}(z)),
$$

where $q$ and $r \in \Omega$ are odd or even, respectively. By (34) and (36) we have

$$
h(0) k(z)=\exp \left(a z^{2}+b z+c+q(z)+\operatorname{ir}(z)\right)
$$

for all complex numbers $z$. By the above equality we have $h(0) \neq 0$. So, setting $m=\frac{1}{h(0)}$ yields

$$
k(z)=m \exp \left(a z^{2}+b z+c+q(z)+\operatorname{ir}(z)\right) .
$$

We have now determined $h$ and $k$ as (32) and (37).

Step 4. We shall prove that

$$
|l m \exp c|=1,
$$

which is a relation between three complex constants $l, m, c$ in (32) and (37). Since $p \in \Omega$ and $s \in \mathfrak{R}, i p(s)$ is purely imaginary. Thus we obtain

$$
|\exp (i p(s))|=1 \text {. }
$$

Since $q \in \Omega$ and $q$ is odd, $q(i t)$ is purely imaginary. So we obtain

$$
|\exp (q(i t))|=1 \text {. }
$$

Since $r \in \Omega$ and $r$ is even, $\operatorname{ir}(i t)$ is purely imaginary. So we obtain

$$
|\exp (\operatorname{ir}(i t))|=1 \text {. }
$$

Substituting (25), (26), (32), and (37) into (5), making some calculations, and using (38), (39), and (40) yield $|l m \exp c|=1$.

\section{AN APPLICATION OF THE MAIN THEOREM}

In this section we shall solve three functional equations, also related to the complex exponential function, by using the main theorem.

We specify how one obtains these equations from (5). From there on the proofs consist of applying Theorem 1. We omit these details. 
Theorem 2. All entire solutions of Hille's functional equation

$$
|f(s+i t)|=|f(s) f(i t)|
$$

are given by

$$
f(z) \equiv 0 \quad \text { and } \quad f(z)=\exp \left(a z^{2}+b z+c\right),
$$

where $a$ is an arbitrary real constant, $b$ is an arbitrary complex constant, and $c$ is an arbitrary purely imaginary constant.

Proof. This is (5) with $g(z) \equiv 1$ and $h(z)=k(z)=f(z)$.

Theorem 3. All entire solutions of the functional equation (4), i.e.,

$$
|f(s+i t) f(s-i t)|=\left|f(s)^{2} f(i t)^{2}\right|
$$

are given by

$$
f(z) \equiv 0 \quad \text { and } \quad f(z)=\exp \left(a z^{2}+b z+c+\operatorname{ir}(z)\right),
$$

where $a, b$ are arbitrary real constants, $c$ is an arbitrary purely imaginary constant, and $r(z)$ is an arbitrary entire even function $\in \Omega$.

Proof. This is (5) with $g(z)=f(z)$ and $h(z)=k(z)=f(z)^{2}$. 22])

Now we consider Lobachevskii's functional equation (cf. [1, p. 7] and [2, p.

$$
f(x+y) f(x-y)=f(x)^{2},
$$

where $f$ is an unknown entire function of a complex variable $z$ and $x, y$ are complex variables. The following theorem holds.

Theorem B. The only entire solutions of (41) are given by

$$
f(z) \equiv 0 \text { and } f(z)=\exp (a z+b),
$$

where $a, b$ are arbitrary complex constants.

If we replace $x$ and $y$ by $s$ and it $(s, t \in \Re)$ in (41) and we take the absolute values of the resulting equality, then we obtain the following functional equation:

$$
|f(s+i t) f(s-i t)|=\left|f(s)^{2}\right|,
$$

where the unknown function $f$ is entire and $s, t$ are real variables.

Theorem 4. All entire solutions of the functional equation (42) are given by

$$
f(z) \equiv 0 \text { and } f(z)=\exp (a z+b+i p(z)),
$$

where $a, b$ are arbitrary complex constants and $p(z)$ is an arbitrary entire function $\in \Omega$.

Proof. The functional equation (42) is (5) with $g(z)=f(z), h(z)=f(z)^{2}$, and $k(z)=1$.

\section{ACKNOWLEDGMENT}

The authors wish to thank Professor J. Aczél and the referee for their many useful suggestions. 


\section{REFERENCES}

1. J. Aczél, Lectures on functional equations and their applications, Academic Press, New York and London, 1966.

2. J. Aczél and J. Dhombres, Functional equations in several variables, Cambridge Univ. Press, Cambridge, New York, and Melbourne, 1989.

3. J. Aczél and H. Haruki, Commentary, Einar Hille's Collected Works (R. R. Kallman, ed.), The MIT Press, Cambridge, Mass. and London, England, 1975, pp. 651-658.

4. J. Aczél, Private communication.

5. $\ldots$ On a generalization of the functional equation of Pexider, Publ. Inst. Math. (Beograd) 4 (1964), 77-80.

6. —, Review for [9], Zbl. 139 (1968), 97.

7. L. V. Ahlfors, Complex analysis, 2nd ed., McGraw-Hill, New York, 1966.

8. R. B. Burckel, An introduction to classical complex analysis, Vol. 1, Academic Press, New York and San Francisco, 1979.

9. H. Haruki, Studies on certain functional equations from the standpoint of analytic function theory, Sci. Rep. Osaka Univ. 14 (1965), 1-40.

10. __ A famous definite integral, Math. Notae 24 (1974), 23-25.

11. __ On a functional equation of Pexider type, Aequationes Math. 36 (1988), 1-19.

12. M. Heins, Complex function theory, Academic Press, New York and London, 1968.

13. E. Hille, A Pythagorean functional equation, Ann. of Math. 24 (1923), 175-180.

14. _ A class of functional equations, Ann. of Math. 29 (1928), 215-222.

15. J. Klippert, A note on an analytic logarithm, Amer. Math. Monthly 88 (1981), 347.

16. Z. Nehari, Introduction to complex analysis, Allyn and Bacon, Boston, Mass., 1969.

17. R. Nevanlinna and V. Paatero, Introduction to complex analysis, Addison-Wesley, Reading, Mass., 1964.

18. A. M. Ostrowski, Über die Funktionalgleichung der Exponentialfunktion und verwandte Funktionalgleichungen, Jber. Deutsch. Math.-Verein 38 (1929), 54-62.

19. R. M. Robinson, A curious trigonometric identity, Amer. Math. Monthly 64 (1957), 83-85.

20. T. Sato, On the functional equality $|f(x+i y)|=|f(x)||f(i y)|$, J. College Arts Sci. Chiba Univ. 4 (1963), 9-10.

Department of Pure Mathematics, University of Waterloo, Waterloo, Ontario, CANADA N2L 3G1

Department of Mathematics, University of La Verne, P.O. Box 51105, Kifissia, Athens, GREECE 14510 